\title{
Author Correction: Reply to: Postbiotics — when simplification fails to clarify
}

Seppo Salminen (D), Maria Carmen Collado, Akihito Endo (D), Colin Hill (D), Sarah Lebeer, Eamonn M. M. Quigley (D), Mary Ellen Sanders, Raanan Shamir, Jonathan R. Swann, Hania Szajewska D and Gabriel Vinderola

Correction to: Nature Reviews Gastroenterology \& Hepatology https://doi.org/10.1038/s41575-021-00522-5, published online 23 September 2021.

In the original Supplementary Table associated with this Correspondence, the terms "postbiotic" and "ISAPP" were misspelled in the column heading and footnote, respectively. These errors have now been corrected and the Supplementary information updated online; for transparency, the updated Supplementary Table is available in the online version of this Correction.

Supplementary information

The online version contains supplementary material available at https://doi.org/10.1038/s41575-022-00596-9.

https://doi.org/10.1038/s41575-022-00596-9 I Published online 1 March 2022

(c) The Author(s), under exclusive licence to Springer Nature Limited 2022 